\title{
Comparison of Colistin MIC by Microbroth Dilution, E-Test and Vitek-2, in Isolates of Acinetobacter spp. Isolated from Bloodstream Infections
}

\author{
Nazir Ahmad Var ${ }^{1}$, Nisar Ahmad Wani ${ }^{2}$, Syed Khurshid Ahmad ${ }^{3}$ \\ ${ }^{1}$ Senior Resident / Demonstrator, ${ }^{2}$ Research Scientist; \\ Department of Microbiology, Government Medical College Baramulla, India, \\ ${ }^{3}$ Professor; Department of Microbiology, SKIMS Medical College Bemina, Srinagar, India.
}

Corresponding Author: Nazir Ahmad Var.

\begin{abstract}
Background: Acinetobacter species are leading cause of nosocomial infections, causing significant morbidity and mortality globally including India. Being persistent in the hospital environment and rapidly developing resistance to a wide variety of antibiotics are the most important features of this pathogen. The present study aimed to compare Colistin MIC of Acinetobacter species isolated from the blood samples by E test and Vitek 2 to the standard broth micro dilution test.
\end{abstract}

Methodology: Two antibiotic susceptibility test methods, The Vitek-2 and the E test, against the reference broth micro dilution method in terms of the various parameters such as Reproducibility, reliability, cost and time effectiveness. Data obtained from the current study regarding antimicrobial resistance of Acinetobacter species recovered from clinical specimens referred to microbiology laboratory of SKIMS and was analyzed by using SPSS 20.0 .

Results: Out of 100 isolates of Acinetobacter species analyzed from blood specimens the distribution of Acinetobacter species according to different clinical diagnosis of patients $89 \%$ were $A$. baumannii and $11 \%$ were $A$. Iwoffii. Seventy three percent of them were from males and $27 \%$ of them were from females with a mean age of 39.6 ( $\mathrm{SD} \pm 27.46$ ). Regarding the specimen and isolate sources, the majority were from ICU (54\%), Surgical ward (26\%), Medical ward (16\%) and $4 \%$ from Outpatient department of SKIMS. Significant descending trends of antimicrobial resistance was shown for Amoxicillin/Clavulanic acid, Cefoperazone/ Sulbactam combination, Cotrimoxazole (100\%), Levofloxacin (92\%) Piperacillin/Tazobactam, Ciprofloxacin (90\%), Cephalosporins (>80\%), Imipenem and Meropenem (76\%), Amikacin (68\%), Gentamycin (67\%), Tigecycline (11\%) and $0 \%$ for Colistin respectively.

Conclusion: from the study it could be concluded that the best reference method for testing susceptibility to the Polymyxins still remains to be defined. However, in routine clinical practice in most regions worldwide, where a reference method can hardly be implemented, the interpretation of Colistin susceptibility should preferably be based on results of automated systems such as Vitek-2 or the E test. The micro broth Dilution method remains the most reliable and reproducible, however most tedious and time-consuming method. Colistin remains a very effective, least resisted drug for MDR Acinetobacter species as compared by all the three methods.

Keywords: Acinetobacter species; Antimicrobial resistance; Colistin; E test and Vitek 2

\section{INTRODUCTION}

Bacterial species belonging to the genus Acinetobacter specially A. baumannii is strictly aerobic undoubtedly the important causative pathogen of a variety of nosocomial infections such as bacteremia, hospital-acquired pneumonia, and urinary 
tract infections besides having emerged as important causative pathogens of ventilatorassociated pneumonia as well. This organism commonly presents resistance to multiple antimicrobial agents, including carbapenems (multidrug resistance) and possesses resistance against broad-spectrum cephalosporins which is due to horizontal transfer of a chromosomal segment containing ISAba1-ampC, $\beta$-lactam agents by forming $\beta$-Lactamase, aminoglycosides and quinolones. Colistin and other polymyxins are cyclic cationic peptides produced by the soil bacterium Bacillus polymyxa that act by disrupting the negatively charged outer membranes of gram-negative bacteria. specific modification of the lipid A component of the outer membrane lipopolysaccharide, resulting in a reduction of the net negative charge of the outer membrane; In the Past few years, Colistin is increasingly being used as a last-resort treatment option for infections caused by MDR organisms (Nation, et al., 2015) particularly carbapenem-resistant (CR) Gram-negative bacteria (Karaiskos et al., 2014). However, an increase in Colistin resistance has emerged worldwide, especially Acinetobacter isolates, further limiting treatment options (Ah et al, 2014; Cai et al., 2014; Sader et al., 2014). Hence the quest for a Rapid and reliable Colistin susceptibility testing (ST) is much needed in routine clinical laboratories to allow appropriate therapeutic decision-making. Till now, not many studies have assessed the performance of Colistin ST methods, displaying controversial results, and thus, the most accurate one is still challenging. The commonly used, Disk diffusion method yielded high error rates compared to MICbased methods and is now considered as unreliable for the detection of Colistin resistance (Gales et al., 2001; Lo-Ten-Foe et al., 2007; Maalej et al., 2011). Among commercial methods, gradient diffusion strips are convenient tests for determining Colistin MICs, but their performance is not well established. Some studies have demonstrated very good correlations between the results of E-test (bioMérieux, Marcy l'Etoile, France) and broth microdilution (BMD) or agar dilution (AD) methods for ColistinST(Lo-Ten-Foe et al., 2007; Maalej et al., 2011; Arroyo et al., 2005; Lee et al., 2013; Goldstein et al., 2007)while other reports have questioned the reliability of E-test (Hindler et al., 2013; Tan et al., 2007). Colistin Susceptibility Testing using automated methods has been tested in a limited number of studies that have tested mainly the performance of the Vitek2 system (bioMérieux)(Lo-Ten-Foe et al., 2007; Lee et al., 2013; Tan et al., 2007.Now-a-days, Agar Dilution and Broth Microdilution are considered the standard methods for MIC Susceptibility Testing. These tests, however, are not convenient for routine clinical laboratories. Additionally, for Broth Micro dilution, technical issues, such as the type or surface pretreatment of microtitre trays, have influenced Colistin MICs significantly (Albur et al., 2014; Nation et al., 2015) In particular, Colistin displays various levels of adherence to different surfaces used for MIC trays, such as polystyrene, resulting in reduced antibiotic concentrations actually being present under experimental conditions (Karvanen et al., 2011). The addition of the surfactant polysorbate 80 (P80) to Broth Microdilution (BMD-P80) has been found to minimize Colistin adhesion to Broth Microdilution panels and thus significantly reducing Colistin MICs, affecting mainly bacteria with relatively low MICs $(\leq 2$ $\mu \mathrm{g} / \mathrm{ml}$ ) (Hindler et al., 2013; Albur et al., 2014; Sader et al., 2012) but, the CLSI does not recommend the use of P80 for Colistin ST by BMD. In addition, according to recent observations, P80 has been found to exhibit a synergistic effect with Colistin, probably enhancing its interaction with the bacterial cell membrane. Hence, further studies on the accuracy of BMD-P80 in determining the Colistin susceptibility of Gram-negative bacteria are needed. The interpretations of antimicrobial Susceptibility testing are further 
complicated by the fact that the breakpoints for Colistin developed by various organizations differ. Diffusion tests are cheap compared to most MIC-determination methods. The E-test is a diffusion test, but has been developed to give an approximate MIC-value. The E-test gradient technology is based on a combination of the concepts of dilution and diffusion principles for susceptibility testing. As with other dilution methods, E-test directly quantifies antimicrobial susceptibility in terms of discrete MIC values. However, in using a predefined, stable and continuous antibiotic concentration gradient, E-test MIC values can be more precise and reproducible than results obtained from conventional procedures based on discontinuous two-fold serial dilutions. The MIC breakpoints for defining interpretive categories are published by the CLSI ${ }^{\circledR}$ and the EUCAST. Being a fully quantitative MIC method, Etest enables the laboratory to report the exact MIC value together with the interpretive category. E-test generates MIC values from a continuous scale and can give results in-between conventional two-fold dilutions i.e. half dilutions. An E-test MIC value which falls between standard two-fold dilutions has to be rounded up to the next upper two-fold value before categorization. The Vitek-2 system is the second generation of Vitek and offers a more sophisticated model of data analysis as well as a fully automated process for card identification, organism suspension dilution, and card filling. Once these steps are complete, the Vitek-2 seals the cards into a chamber to prevent contamination during processing. The cards are then loaded into the reader incubator, which ejects them at the end of testing. The Vitek-2 uses colorimetric technology utilizing three wavelengths of light to provide broad profiles for the most clinically significant organisms. Species identification with these systems is complete in an average of 3 hours with rapid methods, but may take up to 5-7 hours for slow-growing organisms that require colorimetric testing methodology.
Susceptibility results may take up to 15 hours, with a mean of about 9 hours. One major advantage of automated susceptibility methodologies is a reduction in labour (Jossart and Courcol, 1999). Another advantage is that these systems can provide faster reporting of susceptibility results, potentially leading to the earlier initiation of appropriate antibiotic therapy. Rapid bacterial identification and susceptibility testing not only improve patient therapy and outcome but also reduce costs (Doern et al., 1994)Although the reduction in labour requirements and faster reporting are significant advantages to using automation in the Microbiology laboratory, definite disadvantages exist. Automation has a definite place in large Microbiology labs that cater to a large populace but there are reservations to its use in average to small sized laboratory where such a technology would be underutilized. In this study we compared Colistin MIC of Acinetobacter species isolated from the blood samples by $\mathrm{E}$ test and Vitek 2 to the standard Broth Microdilution test.

\section{MATERIALS AND METHODS 2.1 Sample collection}

Over the course of this study (from June 2016 to May 2017) a total of 100 samples were collected from patients in different services like ICU, Medical ward, surgical ward and Outpatient department of Sheri-Kashmir Institute of Medical Sciences (SKIMS) Srinagar, the specimen analyzed for the current study was blood.

\subsection{Isolation and identification of $A$ baumannii}

Positive blood culture samples from Bact /Alert microbial detection system were selected and aliquots from these bottles were gram stained.

\section{2 .1 Process by the Vitek-2 system}

All the strains isolated from blood cultures that were identified by Vitek-2 as Acinetobacter spp. were incorporated in the study. The identification and susceptibility 
testing were done by Vitek-2 facility. The same growth was processed for Microbroth Dilution Method for the antibiotic sensitivity to get its MIC.A Part of the same growth was used for the E-test on Muller Hinton plate and the results of the above methods were compared. Duplicate isolates from the same patient were considered as one. To avoid unnecessary variability in the visual determination of MICs, results were read by the same individual. Discordant results were resolved by repeat testing of all AST methods, including the reference method. Analysis was performed using discordant data that persisted through secondary testing. The Vitek-2 system has been designed to provide Identification/ Antibiotic susceptibility test (ID/AST) results within 5 to 8 hours and the Vitek 2 Compact works with ready-to-use Vitek 2 ID/AST cards. The Suspensions obtained thus were prepared by emulsifying bacterial isolates in $0.45 \%$ saline to the equivalent of a 0.5 McFarland turbidity standard.

\begin{tabular}{|c|c|}
\hline Product & McFarland Turbidity Range \\
\hline GN & $0.50-0.63$ \\
\hline GP & $0.50-0.63$ \\
\hline YST & $1.80-2.20$ \\
\hline BCL & $1.80-2.20$ \\
\hline
\end{tabular}

The same suspension was used for AST on the Vitek-2 system. Suspensions for the comparative identification method were made according to the manufacturer's recommendations.

\subsubsection{Process by the Microbroth dilution test (Reference method)}

(a) Preparation of antibiotic stock solution for Colistin;

Stock solution was prepared using the formula $1000 / P \times V \times C=W$, where $\mathrm{P}=$ potency given by manufacturer $(\mu \mathrm{g} / \mathrm{mg}), \mathrm{V}=$ volume required $(\mathrm{ml}), \quad \mathrm{C}=$ Final concentration of solution $(\mathrm{mg} / \mathrm{L})$ and $\mathrm{W}=$ weight of the antibiotic (mg) to be dissolved in volume $\mathrm{V}(\mathrm{ml})$. The Colistin stock solution was prepared by dissolving 78.7 $\mathrm{mg}$ of the antibiotic solution in $2 \mathrm{ml}$ of distilled water.

\section{(b) Preparation of the working antibiotic solution}

Working solution was prepared as per the formula V1C1=V2C2 (V1 is volume of starting solution needed, $\mathrm{C} 1$ is the concentration of starting solution needed, V2 is the final volume of the new solution and C2 is the final concentration of the new solution) The working solution was prepared one concentration higher than the highest concentration of the drug to be tested. Thus, for Colistin, $1.63 \mu \mathrm{L}$ of working solution was prepared by dissolving $64 \mathrm{mg} / \mathrm{ml}$ of stock solution in Muller Hinton broth.

\subsubsection{Microbroth dilution method}

Using a micropipette $100 \mu \mathrm{l}$ of Muller Hinton broth was dispensed into all wells of a micro titer plate leaving the first well unfilled. In the first well, $200 \mu \mathrm{L}$ of MH broth was poured. After this, $1.63 \mu \mathrm{l}$ of working antibiotic was added to this well making the concentration to $64 \mu \mathrm{g} / \mathrm{ml}$. From the first well, $100 \mu \mathrm{l}$ of the working antibiotic solution was taken. It was mixed six to eight times, pipetted out and added to the second well, already containing $100 \mu \mathrm{l}$ of $\mathrm{MH}$ broth. So, the final concentration in the first well was $32 \mu \mathrm{g} / 100 \mathrm{ml}$. From the Second well, $100 \mu \mathrm{l}$ of solution was added into the next well and so on and so forth till the well no.10 was reached from which $100 \mu \mathrm{l}$ of solution was discarded. The final concentration in the wells ranged from $0.06 \mu \mathrm{g} / \mathrm{ml}$. The last two columns served as growth control and media control respectively. The turbidity of the bacterial inoculum was adjusted to 0.5 McFarland standards and $5 \mu \mathrm{l}$ of it was dispensed into all wells of micro titer plate except 12 well. The plate was incubated at 37 degrees Celsius overnight and read the other day. The results were recorded by the visual inspection of microtiter plate at overnight incubation at 37 degrees as per CLSI guidelines.

\subsubsection{Process by E-test}

For the E-Test 90-mm-diameter Mueller-Hinton agar plates were inoculated 
with swabs saturated with suspensions of the study isolates equivalent to a 0.5 McFarland standard. The Colistin-coated test strips were placed in the plate in accordance with the manufacturer's instructions. The antimicrobial concentration ranges tested were noted. E test strips were applied when the agar surface was completely dry. The results were read after 16 to 20 hrs of incubation in ambient air at $35.8^{0} \mathrm{C}$. The MIC was determined at the point where inhibition of the growth intersected with E-test strip. If Etest's results were between twofold dilutions, they were rounded to the next highest twofold dilution. The susceptibility test results were interpreted according to CLSI breakpoint recommendations in the Etest, Vitek 2 and broth micro dilution methods, $\leq 2 \mathrm{mg} / \mathrm{l}$ and $\geq 4 \mathrm{mg} / \mathrm{l}$ were accepted as sensitive and resistant, respectively for Acinetobacter isolates.

\subsection{Preservation of Strains}

The clinical isolates identified were preserved in $15 \%$ glycerol-normal saline solution and stored at $-20^{\circ} \mathrm{C}$. Fresh cultures were prepared from these stock cultures as and when required. A Comparison between the two test methods as compared to the reference method was then done regarding reproducibility, reliability, cost and time effectiveness.

\subsection{Statistical Analysis}

The recorded data was compiled and entered in a spreadsheet (Microsoft Excel) and then exported to data editor of SPSS Version 20.0 (SPSS Inc., Chicago, Illinois, USA). Continuous variables were summarized in the form of means and standard deviations and categorical variables were summarized as percentages. Analysis of variance (ANOVA) was employed to compare the time effectiveness of various AST methods. A P-value of less than 0.05 was considered statistically significant. Reproducibility was assured by the repeat testing of all AST methods, including the reference method. Analysis was performed using discordant data that persisted through secondary testing.

\section{RESULTS}

A total of 100 blood stream isolates confirmed by Vitek 2 as Acinetobacter spp. were included in the study. Of the 100 isolates included in the study, $24 \%$ of the isolates belonged to children $<10$ years of age, followed by 11 (11\%) patients were aged 10-29 years, 19 (19\%) were 30-49 years old, 31 (31\%) studied patients belonged to age group of between 50 and 69 years while 15 (15\%) belonged to patients in the age group above 70 years (Table 1; Fig 1). $73 \%$ of the isolates belonged to Male patients and $27 \%$ to female patients (Table 2; Fig 2).54\% of the isolates belonged to ICU patients where as $26 \%$ to the surgical wards. $16 \%$ of the isolates obtained, were from the Medical wards and 4\% from OPD (Table 3; Fig 3). Of the 100 isolates identified by Vitek-2, $89 \%$ were Acinetobacter baumannii and $11 \%$ were Acinetobacter lwoffii (Table 4; Fig 4).Out of the 100 isolates tested $100 \%$ resistance was observed against Amoxicillin/Clavulanic acid.90\% of the isolates demonstrated resistance to Piperacillin/Tazobactam. These isolates exhibited a high degree of resistance to cephalosporins $>80 \%$ whereas a $100 \%$ resistance was observed for Cefoperazone/ sulbactam combination (Table 5A; Fig $5 \mathrm{~A}) .76 \%$ of the isolates were found to be resistant to carbapenems (Table 5b; Fig 5b). Among Quinolones $>90 \%$ isolates were resistant against both Ciprofloxacin and Levofloxacin. Among the Aminoglycosides, resistance to Amikacin and Gentamycin was found to be $67 \%$ and $68 \%$ respectively (Table 5c; Fig 5c). In other classes of drugs, $100 \%$ were resistant to Cotrimoxazole.11\% of the isolates showed resistance to Tigecycline however none of the isolates were found to be resistant to Colistin (Table 5d; Fig 5d). Out of the 100 isolates tested for Acinetobacter spp. by E Strip, $86 \%$ had a MIC of $0.5-1.0 \mu \mathrm{g} / \mathrm{ml}$ while $13 \%$ had a MIC of $1.0-1.5 \mu \mathrm{g} / \mathrm{ml}$ for Colistin. $1 \%$ of the isolates were found to have a MIC of $>4$ for Colistin (Table 6;Fig 6). Out of the 100 
Nazir Ahmad Var et.al. Comparison of Colistin MIC by Microbroth dilution, E-test and Vitek-2, in isolates of Acinetobacter spp. isolated from bloodstream infections

isolates tested for Acinetobacter spp. by Vitek 2, $100 \%$ had a MIC of $\leq 0.5 \mu \mathrm{g} / \mathrm{ml}$ for Colistin (Table7;Fig7).Out of the 100 isolates tested for Acinetobacter spp. by Broth Micro dilution, 78\% had a MIC $\leq 0.5$ $\mu \mathrm{g} / \mathrm{ml}$ while $22 \%$ had a MIC of $0.5-1.0$ $\mu \mathrm{g} / \mathrm{ml}$ for Colistin (Table 8; Fig 8). $100 \%$ of the isolates tested by Vitek-2 were in categorical agreement with Broth micro dilution whereas $99 \%$ of the Isolates tested by E-test were also in categorical agreement with the reference method, and the incidence of a major error by $\mathrm{E}$ test was only $1 \%$. No Very major errors (VMEs) were found with any of the tested methods (Table 9). The time required for Antibiotic susceptibility testing by the various methods was, Broth Micro dilution (22.1 hrs) >E-test (17.8 hrs) >Vitek 2(8.7 hrs) and the difference was statistically significant (p value $<0.001)$ ( Table 10; Fig 9).The cost in Rupees per Isolate per one test for Antibiotic susceptibility testing by the various methods was, Vitek 2(Rs 243) $>$ E test (Rs 100) > Micro broth Dilution (Rs 20) (Table 11; Fig 10).

\section{Supplementary File- 01}

Table 1: Age wise distribution of Acinetobacter isolates

\begin{tabular}{|c|c|c|}
\hline Age (years) & Frequency & Percentage \\
\hline$<10$ & 24 & $24 \%$ \\
\hline $10-29$ & 11 & $11 \%$ \\
\hline $30-49$ & 19 & $19 \%$ \\
\hline $50-69$ & 31 & $31 \%$ \\
\hline$\geq 70$ & 15 & $15 \%$ \\
\hline Total & $\mathbf{1 0 0}$ & $\mathbf{1 0 0} \%$ \\
\hline \multicolumn{3}{|c|}{ Mean \pm SD $=\mathbf{3 9 . 6} \pm \mathbf{2 7 . 4 6}$} \\
\hline
\end{tabular}

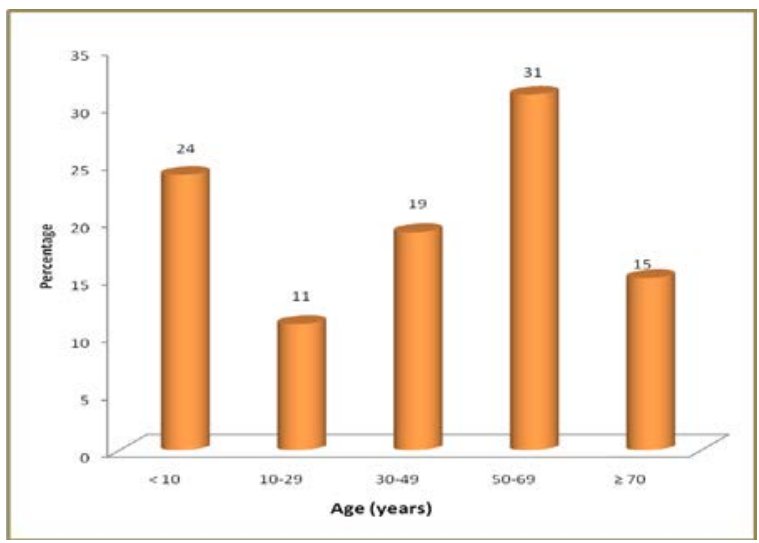

Fig. 1: Bar Diagram showing Age wise Distribution of Acinetobacter isolates

Table 2: Gender distribution of Patients from whom Acinetobacter spp. were isolated

\begin{tabular}{l|l|l} 
Gender & Frequency & Percentage \\
\hline
\end{tabular}

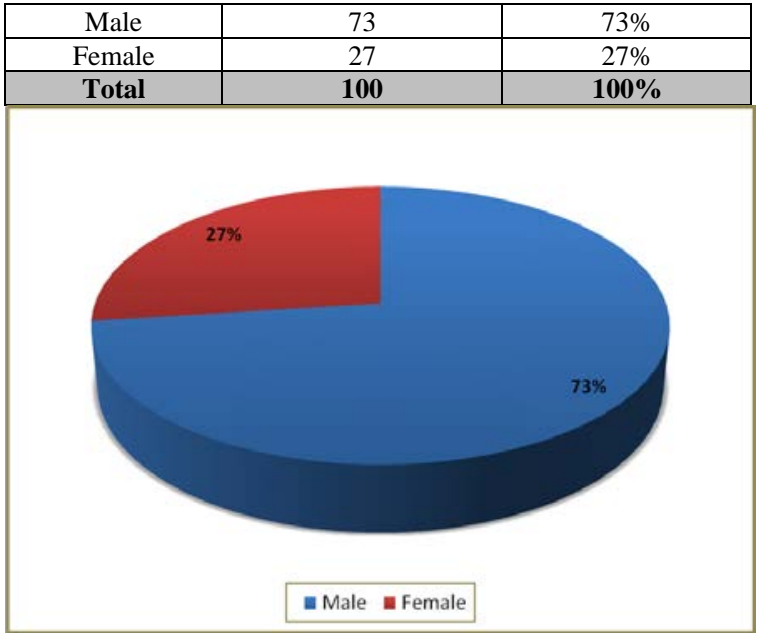

Fig. 2: Pie Chart Showing Gender distribution of Patients with Acinetobacterinfection

Table 3: Ward wise distribution Acinetobacterisolates

\begin{tabular}{|c|c|c|}
\hline Ward & Frequency & Percentage \\
\hline ICU & 54 & $54 \%$ \\
\hline Medical & 16 & $16 \%$ \\
\hline Surgical & 26 & $26 \%$ \\
\hline OPD & 4 & $4 \%$ \\
\hline Total & $\mathbf{1 0 0}$ & $\mathbf{1 0 0} \%$ \\
\hline
\end{tabular}

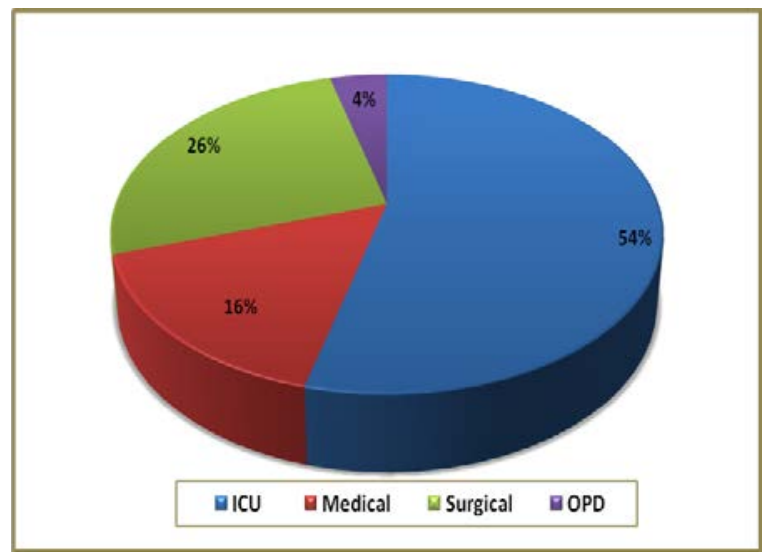

Fig. 3: Pie chart showing ward wise distribution of Acinetobacter isolates.

Table 4: Species distribution of Acinetobacter isolates.

\begin{tabular}{|c|c|c|}
\hline Acinetobacter Species & Frequency & Percentage \\
\hline Acinetobacter baumannii & 89 & $89 \%$ \\
\hline Acinetobacter Lwoffii & 11 & $11 \%$ \\
\hline Total & $\mathbf{1 0 0}$ & $\mathbf{1 0 0 \%}$ \\
\hline
\end{tabular}

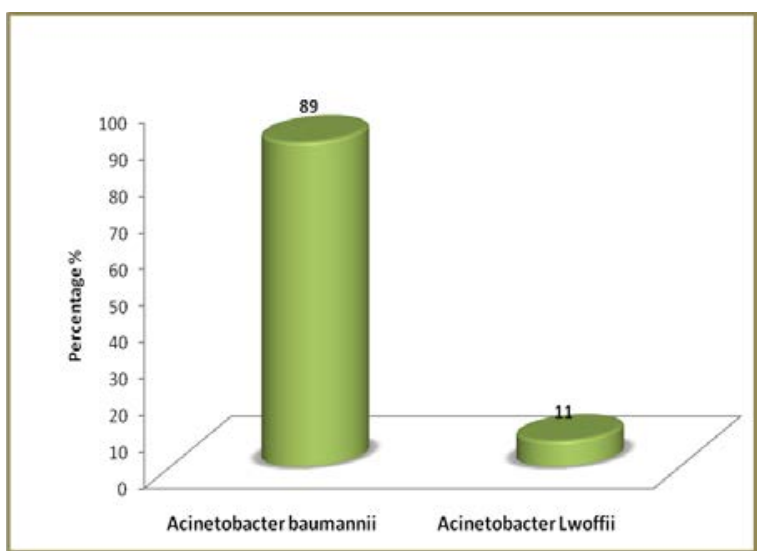


Nazir Ahmad Var et.al. Comparison of Colistin MIC by Microbroth dilution, E-test and Vitek-2, in isolates of Acinetobacter spp. isolated from bloodstream infections

Fig. 4: Bar Diagram showing the percentage of different Acinetobacter spp. isolated from the study patients. Table 5a: Vitek-2 antibiotic susceptibility pattern of Acinetobacter isolates

\begin{tabular}{|l|l|c|c|}
\hline \multicolumn{2}{|l|}{ Drug } & Frequency & Percentage \\
\hline Amoxicillin/ & Resistant & 100 & $100 \%$ \\
\cline { 2 - 4 } Clavulanic acid & Sensitive & 0 & $0 \%$ \\
\cline { 2 - 4 } & Intermediate Sensitive & 0 & $0 \%$ \\
\hline \multirow{3}{*}{$\begin{array}{l}\text { Piperacillin/ } \\
\text { Tazobactam }\end{array}$} & Resistant & 90 & $90 \%$ \\
\cline { 2 - 4 } & Sensitive & 10 & $10 \%$ \\
\cline { 2 - 4 } & Intermediate Sensitive & 0 & $0 \%$ \\
\hline \multirow{3}{*}{ Cefuroxime } & Resistant & 99 & $99 \%$ \\
\cline { 2 - 4 } & Sensitive & 1 & $1 \%$ \\
\cline { 2 - 4 } & Intermediate Sensitive & 0 & $0 \%$ \\
\hline \multirow{3}{*}{ Cefepime } & Resistant & 86 & $86 \%$ \\
\cline { 2 - 4 } & Sensitive & 4 & $4 \%$ \\
\cline { 2 - 4 } & Intermediate Sensitive & 10 & $10 \%$ \\
\cline { 2 - 4 } & Resistant & 3 & $83 \%$ \\
\cline { 2 - 4 } & Sensitive & 15 & $15 \%$ \\
\hline \multirow{2}{*}{$\begin{array}{l}\text { Cefoperazone/ } \\
\text { Sulbactam }\end{array}$} & Resistant & 82 & $2 \%$ \\
\cline { 2 - 4 } & Sensitive & 13 & $13 \%$ \\
\cline { 2 - 4 } & Intermediate Sensitive & 2 & $2 \%$ \\
\hline
\end{tabular}

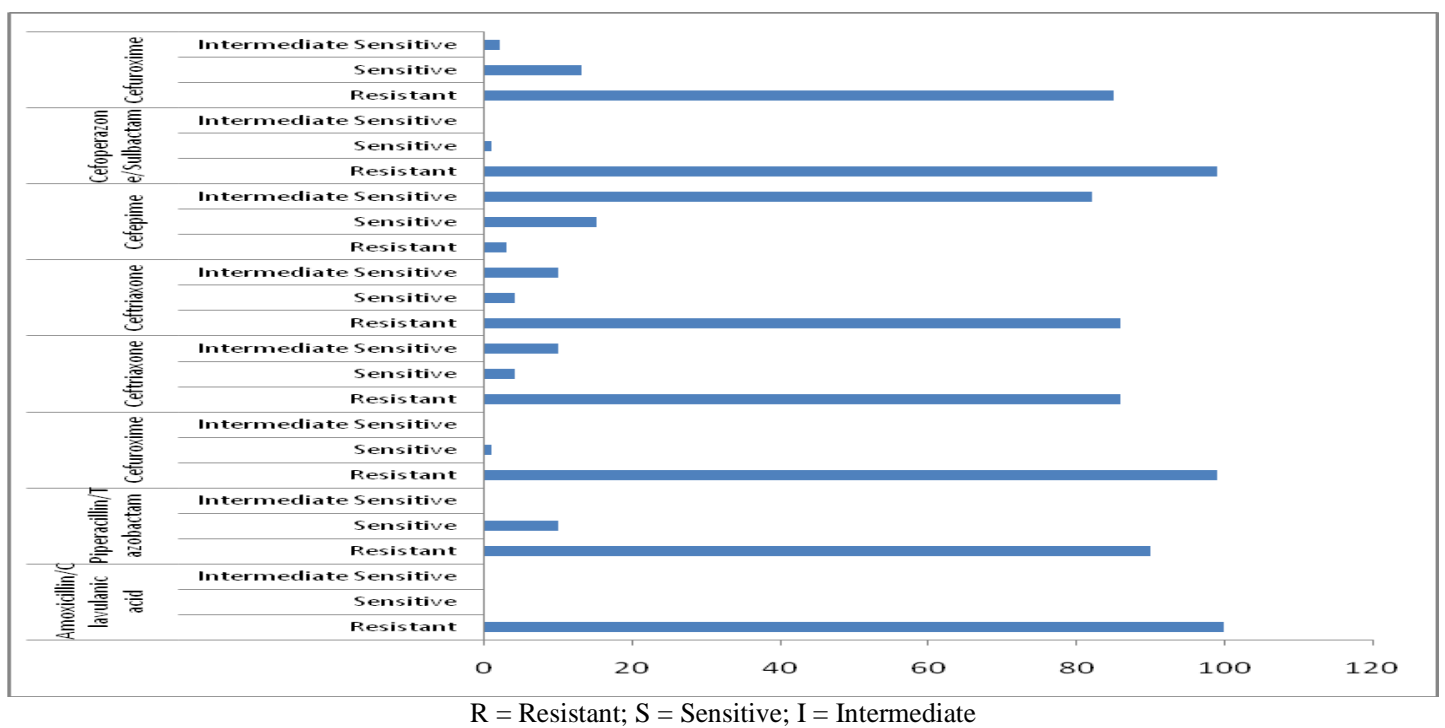

Fig. 5a: Bar chart depicting the antibiotic Susceptibility Profile of Acinetobacter isolates by Vitek 2

Table 5b: Antibiotic Susceptibility Profile of Acinetobacter spp. Blood stream isolates by Vitek-2

\begin{tabular}{|l|l|c|c|}
\hline \multicolumn{2}{|c|}{ Drug } & Frequency & Percentage \\
\hline \multirow{3}{*}{ Imipenem } & Resistant & 76 & $76 \%$ \\
\cline { 2 - 4 } & Sensitive & 23 & $23 \%$ \\
\cline { 2 - 4 } & Intermediate Sensitive & 1 & $1 \%$ \\
\hline \multirow{2}{*}{ Meropenem } & Resistant & 76 & $76 \%$ \\
\cline { 2 - 4 } & Sensitive & 23 & $23 \%$ \\
\cline { 2 - 4 } & Intermediate Sensitive & 1 & $1 \%$ \\
\hline
\end{tabular}

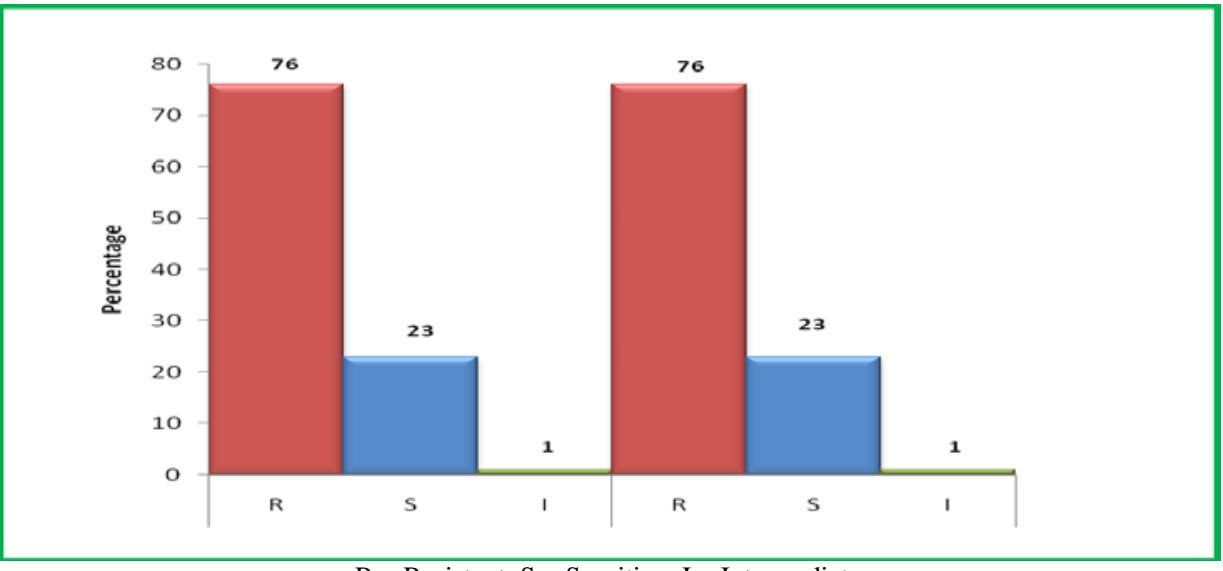

$\mathrm{R}$ = Resistant; S = Sensitive; I = Intermediate 
Nazir Ahmad Var et.al. Comparison of Colistin MIC by Microbroth dilution, E-test and Vitek-2, in isolates of Acinetobacter spp. isolated from bloodstream infections

Fig. 5b: Bar chart depicting the antibiotic Susceptibility Profile of Acinetobacter isolates by Vitek-2, contd. Table 5c: Antibiotic Susceptibility Profile of Acinetobacter spp. Blood stream isolates by Vitek-2

\begin{tabular}{|l|l|c|c|}
\hline \multirow{3}{*}{ Amikacin } & Drug & Frequency & Percentage \\
\cline { 2 - 4 } & Resistant & 68 & $68 \%$ \\
\cline { 2 - 4 } & Sensitive & 23 & $23 \%$ \\
\hline \multirow{3}{*}{ Gentamycin } & Resistant & 9 & $9 \%$ \\
\cline { 2 - 4 } & Sensitive & 67 & $67 \%$ \\
\cline { 2 - 4 } & Intermediate Sensitive & 9 & $24 \%$ \\
\hline Ciprofloxacin & Resistant & 90 & $90 \%$ \\
\cline { 2 - 4 } & Sensitive & 5 & $5 \%$ \\
\cline { 2 - 4 } & Intermediate Sensitive & 5 & $5 \%$ \\
\hline Levofloxacin & Resistant & 92 & $92 \%$ \\
\cline { 2 - 4 } & Sensitive & 5 & $5 \%$ \\
\cline { 2 - 4 } & Intermediate Sensitive & 3 & $3 \%$ \\
\hline
\end{tabular}

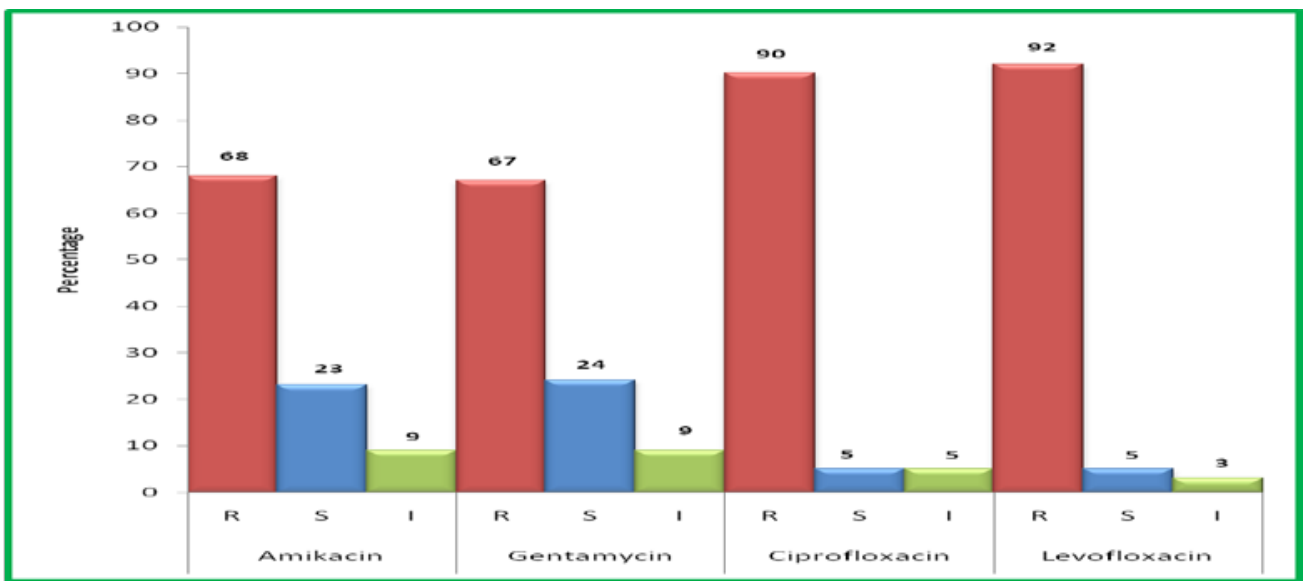

$\mathrm{R}$ = Resistant; $\mathrm{S}$ = Sensitive; I = Intermediate

Fig. 5c: Bar chart depicting the antibiotic Susceptibility Profile of Acinetobacter isolates by Vitek-2, contd.

Table 5d: Antibiotic Susceptibility Profile of Acinetobacter spp. Blood stream isolates by Vitek-2

\begin{tabular}{|l|l|c|c|}
\hline \multicolumn{2}{|l|}{ Drug } & Frequency & Percentage \\
\hline \multirow{3}{*}{ Cotrimoxazole } & Resistant & 100 & $100 \%$ \\
\cline { 2 - 4 } & Sensitive & 0 & $0 \%$ \\
\cline { 2 - 4 } & Intermediate Sensitive & 0 & $0 \%$ \\
\hline \multirow{3}{*}{ Coligecycline } & Resistant & 11 & $11 \%$ \\
\cline { 2 - 4 } & Sensitive & 87 & $87 \%$ \\
\cline { 2 - 4 } & Intermediate Sensitive & 2 & $2 \%$ \\
\cline { 2 - 4 } & Resistant & 0 & $0 \%$ \\
\cline { 2 - 4 } & Sensitive & 100 & $100 \%$ \\
\cline { 2 - 4 } & Intermediate Sensitive & 0 & $0 \%$ \\
\hline
\end{tabular}

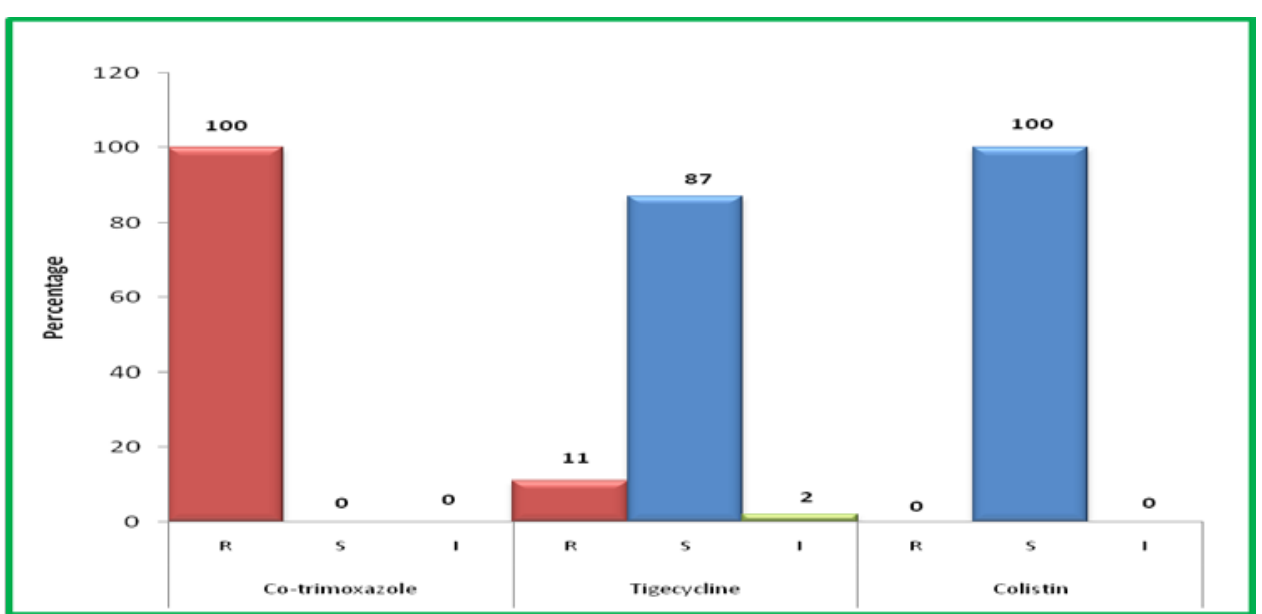

Fig. 5d: Bar chart depicting the antibiotic Susceptibility Profile of Acinetobacter isolates by Vitek-2, contd. 
Nazir Ahmad Var et.al. Comparison of Colistin MIC by Microbroth dilution, E-test and Vitek-2, in isolates of Acinetobacter spp. isolated from bloodstream infections

Table 6: E-test MIC of Colistin by for Acinetobacter isolates

\begin{tabular}{|c|c|c|}
\hline (MIC in $\boldsymbol{\mu g} / \mathbf{m l})$ & Frequency & Percentage \\
\hline $0.25-0.5$ & 0 & $0 \%$ \\
\hline $0.5-1.0$ & 86 & $86 \%$ \\
\hline $1.0-1.5$ & 13 & $13 \%$ \\
\hline$>4$ & 1 & $1 \%$ \\
\hline Total & $\mathbf{1 0 0}$ & $\mathbf{1 0 0} \%$ \\
\hline
\end{tabular}

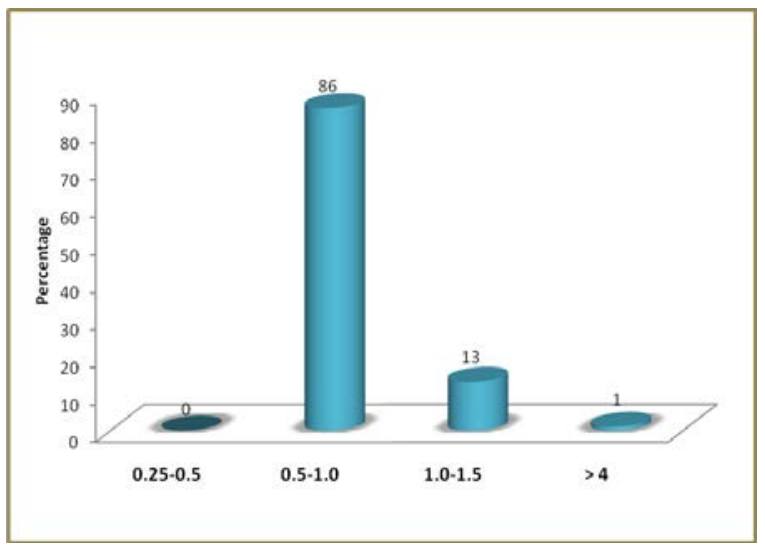

Fig. 6: Bar Diagram to show MIC of Colistin for Acinetobacter spp. by E test.

Table 7: Vitek-2 MIC of Colistin for Acinetobacter isolates.

\begin{tabular}{|c|c|c|}
\hline (MIC in $\mathbf{~ g} / \mathbf{m l})$ & Frequency & Percentage \\
\hline $0.25-0.5$ & 100 & $100 \%$ \\
\hline $0.5-1.0$ & 0 & $0 \%$ \\
\hline $1.0-1.5$ & 0 & $0 \%$ \\
\hline$>1.5$ & 0 & $0 \%$ \\
\hline Total & $\mathbf{1 0 0}$ & $\mathbf{1 0 0} \%$ \\
\hline
\end{tabular}

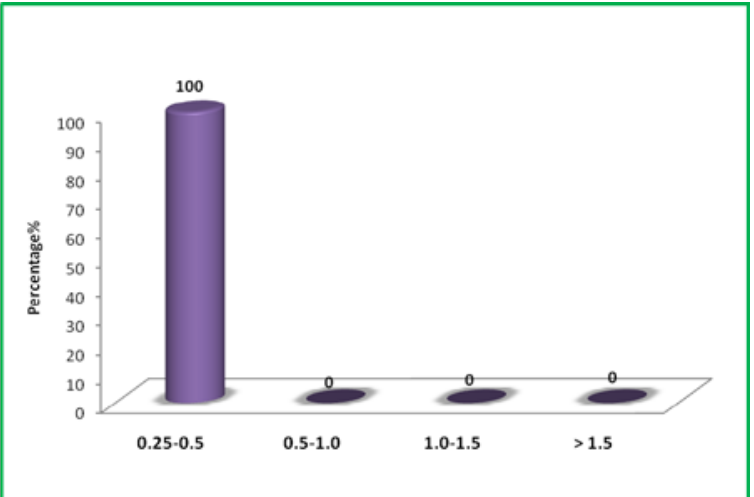

Fig. 7: Bar diagram to show MIC of Colistin for Acinetobacter isolates by Vitek-2

Table 8: Broth Micro dilution MIC of Colistin for Acinetobacter isolates.

\begin{tabular}{|c|c|c|}
\hline (MIC in $\mathbf{~ g} / \mathbf{m l})$ & Frequency & Percentage \\
\hline $0.25-0.5$ & 78 & $78 \%$ \\
\hline $0.5-1.0$ & 22 & $22 \%$ \\
\hline $1.0-1.5$ & 0 & $0 \%$ \\
\hline$>1.5$ & 0 & $0 \%$ \\
\hline Total & $\mathbf{1 0 0}$ & $\mathbf{1 0 0} \%$ \\
\hline
\end{tabular}

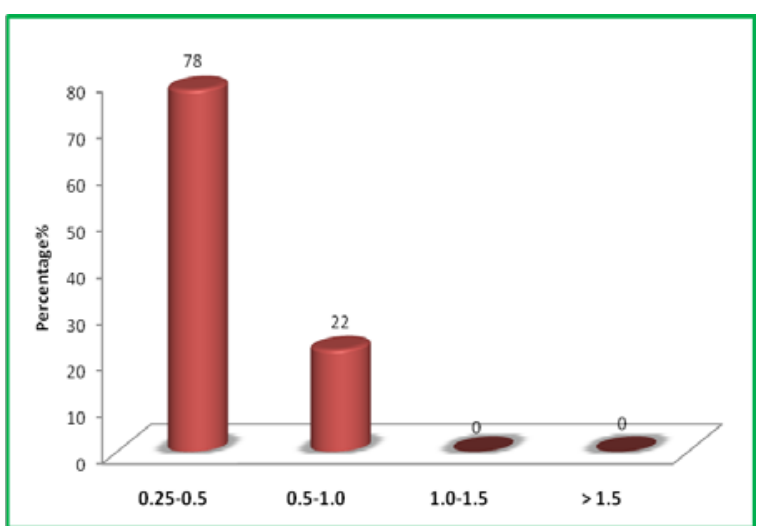

Fig. 8: Bar diagram to show MIC of Colistin for Acinetobacter isolates. by Broth Micro dilution.

Table 9: Comparison of Colistin MIC by E-test and Vitek-2 against the reference i.e. Broth micro dilution method for Acinetobacter isolates.

\begin{tabular}{|l|c|c|c|c|c|}
\hline \multirow{2}{*}{ Testing Method } & \multicolumn{2}{|c|}{ Colistin MIC ( $\mathbf{\mu g} / \mathbf{d l})$} & \multicolumn{2}{c|}{ No. (\%) of isolates } & \multirow{2}{*}{ CA (\%) } \\
\cline { 2 - 5 } & $\mathbf{5 2}$ & $\mathbf{2}$ & $\mathbf{4}$ & $\mathbf{R}$ & \\
\hline Micro Broth Dilution & 100 & 0 & 100 & 0 & $100 \%$ \\
\hline Vitek-2 & 100 & 0 & 100 & 0 & $100 \%$ \\
\hline E-test & 99 & 1 & 99 & 1 & $99 \%$ \\
\hline
\end{tabular}

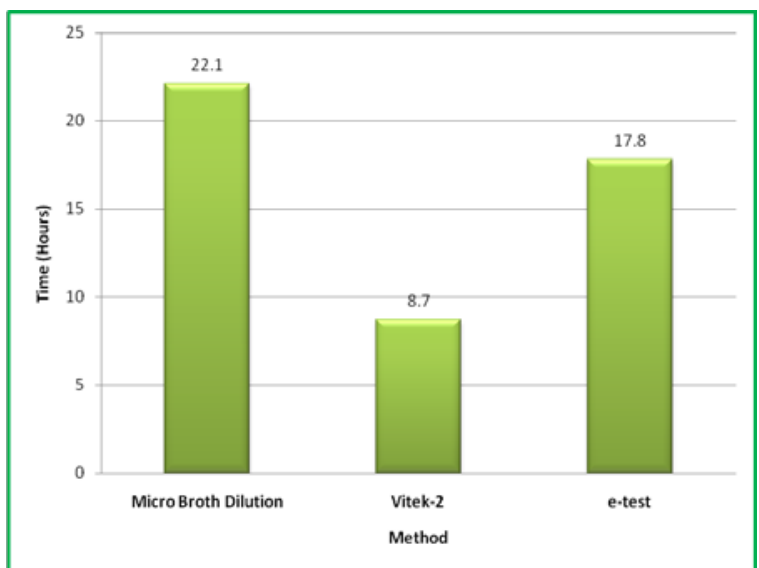

Table 10: Time taken in hours by various methods

\begin{tabular}{|l|c|c|c|c|}
\hline \multicolumn{1}{|c|}{ Testing Method } & Mean & SD & Range & P-value \\
\hline Micro Broth Dilution & 22.1 & 5.37 & $20-24$ & \\
\cline { 1 - 4 } Vitek-2 & 8.7 & 3.25 & $8-9$ & \multirow{2}{*}{$<0.001^{*}$} \\
\hline e-test & 17.8 & 4.58 & $16-20$ & \\
\hline
\end{tabular}

*Statistically Significant Difference (P-value $<0.05)$

Table 11: Cost effectiveness of E-test and Vitek 2 as compared with Broth Micro dilution per Sample.

\begin{tabular}{|c|c|}
\hline Testing Method & Rate (Rs) \\
\hline Micro Broth Dilution & 20 \\
\hline E-test & 100 \\
\hline Vitek-2 & 243 \\
\hline
\end{tabular}

Fig. 9: Bar chart showing Time effectiveness of the various methods in the study group 


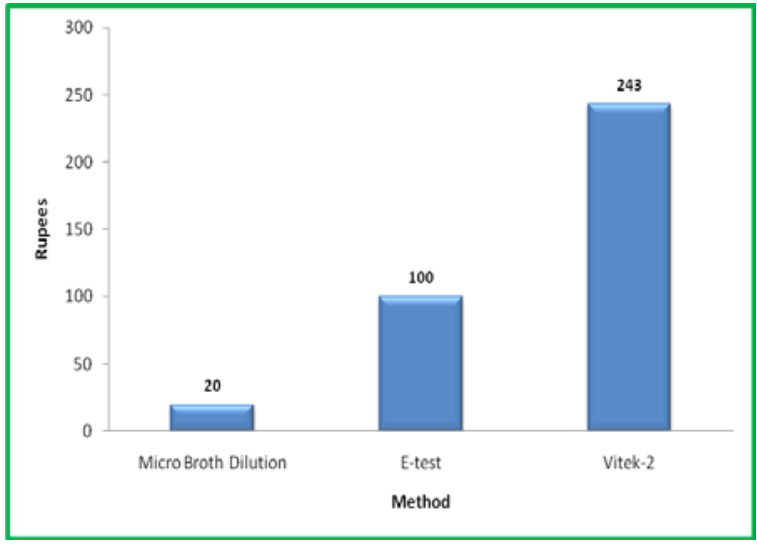

Fig. 10: Bar chart showing Cost effectiveness of the various AST methods in the study groups

\section{DISCUSSION}

In the last few decades Acinetobacter species has emerged as an important nosocomial pathogen, particularly for patients admitted to an intensive-care unit (Gonzalez- Villoria and ValverdeGarduno, 2016). Treatment of these infections is often difficult, because clinical isolates of Acinetobacter species are generally multi drug resistant (Nwadike et al., 2014). Previously the clinical use of polymyxins was limited to topical formulations for the treatment of several diseases, and they were used for the prevention of infection in neutropenic or cystic fibrosis patients. Recently, the potential therapeutic indication for the parenteral use of polymyxins has been restored for the treatment of multidrugresistant Acinetobacter species isolates causing life-threatening infections (Walkty and De-Corby, 2009 ).The in vitro and in vivo activities of Colistin (polymyxin E) have shown it to be an effective antimicrobial agent against Acinetobacter species. However, some polymyxinresistant strains have also been reported (Landman et al., 2008). A few Problems in performing in vitro Colistin susceptibility testing of this organism have also been encountered. The disk diffusion method has been found to be inaccurate and not reproducible for the testing of $A$. baumannii. Agar dilution and broth micro dilution (BMD) are the recommended susceptibility test methods for this organism, but they are cumbersome to perform and impractical to implement as routine tests in many clinical laboratories (Dafopoulou et al., 2015).The E-test and the automated Vitek-2 systems have been found to be simple and accurate alternative methods for determining the antimicrobial susceptibilities of various microorganisms including Acinetobacter species (Ligozzi et al., 2002) However most of the studies have been restricted to Acinetobacter baumannii only. In our study we included all Acinetobacter spp. isolated from blood samples received in our lab. Besides Acinetobacter baumannii some isolates were also identified as Acinetobacter lwoffii although, not many cases of these organisms were detected. Our study is first of its kind in our region to evaluate multiple parameters like reproducibility, reliability, the cost and time effectiveness of the E-test, and Vitek-2 and compared it to that of the BMD reference method, for susceptibility testing of Acinetobacter species to Colistin. Of the 100 isolates included in the study $31 \%$ were isolated from the patients in the age group between 50 and 69 years while 15\% belonged to the age group above 70 years thereby suggesting the higher rate of infections caused by Acinetobacter species in the elderly age group ( $>50$ years). This finding was in concordance with the study by Roohi et al (2017) who found a preponderance (23\%) of Acinetobacter spp. induced infections in elderly patients admitted in the ICU.73\% of the isolates in our study groups belonged to Male patients and $27 \%$ to female patients. Bhattacharyya $S$ et al (2013) reported a similar male preponderance. The gender ratio in their study was 1.46:1 (Male: Female) which was in concordance with our study (Bhattacharyya et al., 2013). In a study by Huidrom $\mathrm{H}$ et al (2015) $66.1 \%$ of Acinetobacter spp. isolated was from males and $33.9 \%$ was from females Similar results were found in studies by Roohi et al (2017) who found a male preponderance. Of the 100 isolates, the majority i.e. $54 \%$ belonged to the Patients admitted to the ICU while the least number of samples i.e. $4 \%$ belonged to 
patients who had visited on OPD basis. Similar results were seen in a study by Victor Ugochukwu Nwadike et al in 2014 that isolated the maximum number of Acinetobacter species from 400 samples in the ICU. Roohi et al., (2017) found similar results in their studies with an increasing occurrence of infections due to Acinetobacter species in ICU patients. Our study was also in concordance with that of Amina Kandeel et al 2016 who demonstrated the presence of Acinetobacter species in different wards, $60 \%$ of which were from the ICU. In our study $89 \%$ of the isolates belonged to the Acinetobacter baumannii species while the remaining $11 \%$ belonged to Acinetobacter Iwoffii species. Bhattacharyya S et al (2013) studied 100 isolates, out of which 54\% (54 isolates) belonged to the $A$. baumannii group, 44\% (44 isolates) were $A$. Iwoffii and 2\% (2 isolates) were identified as $A$. hemolyticus which was in agreement to our findings. Results similar to our study were noted by Lo-Ten-Foe JR.et al in 2006 who obtained 7 species of Acinetobacter baumannii as compared to only 1 specimen of Acinetobacter lwoffii in their study comparing Antibiotic susceptibility testing methods in hetero resistant gram-negative bacteria (Jerome et al., 2007). In our study Colistin demonstrated $100 \%$ activity against Acinetobacter baumannii isolates. This observation was in concordance with the study by Duenas-Diez et al in 2004 who found that Colistin had excellent bactericidal activity against most gramnegative aerobic bacilli, including Acinetobacter species. Our study was also in agreement with a study done by Kandeel et al., in 2016 that demonstrated the highest activity of Colistin against Acinetobacter baumannii isolates as $97 \%$ sensitive. In a study done in Kuwait Sweih et al in 2011 reported Colistin resistance in Acinetobacter baumannii at $12 \%$. In our study tigecycline demonstrated good activity against Acinetobacter species (87\%). Somily et al in their study in 2012demonstrated that $89.3 \%$ of Acinetobacter baumannii strains were susceptible to tigecycline while Kandeel et al. 2016 found $85 \%$ of the isolated Acinetobacter baumannii to be sensitive to tigecycline. Both these studies were in concordance with ours. The Colistin MIC at which $86 \%$ and $14 \%$ of the isolates were inhibited by $E$ test were between 0.5 and 1 $\mu \mathrm{g} / \mathrm{ml}$ and 1 to $1.5 \mu \mathrm{g} / \mathrm{ml}$ respectively while none of the isolates were inhibited below $0.5 \mu \mathrm{g} / \mathrm{ml}$. This correlated poorly with Broth Micro dilution MICs where the Colistin MIC at which $78 \%$ and $22 \%$ of the isolates inhibited by Broth Micro dilution test was below $0.5 \mu \mathrm{g} / \mathrm{ml}$ and 0.5 to $1 \mu \mathrm{g} / \mathrm{ml}$ respectively. This is in concordance to the study by L. A Arroyo et al in 2005in which Colistin MICs at which $50 \%$ and $90 \%$ of isolates were inhibited were 0.06 and 32 $\mu \mathrm{g} / \mathrm{ml}$, respectively, by BMD and 0.5 and $16 \mu \mathrm{g} / \mathrm{ml}$, respectively, by E-test. The agreement within 1 twofold dilution between the E-test and the BMD reference method was $16.5 \%$ (19 of 115 strains). The worst agreement was found mainly with strains for which MICs ranged from 0.06 to $0.25 \mu \mathrm{g} / \mathrm{ml}$ by the reference method. This fact could be related to the poor diffusion of polymyxins in agar (Gales et al., 2001). In a study by Janet A. Hindler et al in 2013it was noted that at susceptible concentrations less than $4.0 \mu \mathrm{g} / \mathrm{ml}$ in the 107 isolates, the Colistin MICs measured by the E test were significantly elevated compared to those measured by BMD test (average MIC, 1.0 versus $0.5 \mu \mathrm{g} / \mathrm{ml}$ by BMD Testing) which was found to be statistically significant. This again was in concordance with our study. However, in our Study, one major error but no Very major errors (VMEs) were noted in the susceptibility testing by E test which was closely in concordance with the study by Jerome R. Lo-Ten-Foe et al in 2006 who compared VITEK 2, Disk Diffusion, E-test, Broth Micro dilution, and Agar Dilution Susceptibility Testing Methods for Colistin in Clinical Isolates, Including Hetero resistant Enterobacter cloacae and Acinetobacter baumannii Strains. The comparison of the E-test method to the reference broth micro dilution 
method showed relatively high levels of agreement. The E-test on IsoSensitest agar was found to be a more sensitive method to detect resistant sub populations. However, no VME was found with the Acinetobacter baumannii strains. Similar results were given by Tan et al in 2006in their study that compared E test, Vitek and Agar dilution for susceptibility testing of Colistin. Out of the 58 isolates of Acinetobacter species tested Major errors were found in $1 \%$ whereas none of the isolates had VMEs. In a study conducted by Lee et al in 2013at a Korean university hospital which compared Vitek 2, Micro scan and E test Methods with the Agar Dilution Method in Assessing Colistin Susceptibility of Bloodstream Isolates of Acinetobacter species, no VMEs were found in the 213 isolates by $\mathrm{E}$ test while the number of major errors was also low at $0.9 \%$.Some discordance exists with the study conducted by K. Dafopoulou et al., in 2015who found that the E test yielded the highest rates of VMEs overall (39.3\%) in a study evaluating Colistin Susceptibility Testing Methods among Carbapenem-non susceptible Klebsiella pneumoniae and Acinetobacter baumannii Clinical Isolates. It could be attributed to the fact that their study was limited to carbapenem resistant Species of Acinetobacter. The Colistin MIC at which $100 \%$ of the isolates were inhibited by Vitek 2 was less than $0.5 \mu \mathrm{g} / \mathrm{ml}$. This correlated well with Broth Micro dilution method where the Colistin MIC at which $78 \%$ of the isolates inhibited was also below $0.5 \mu \mathrm{g} / \mathrm{ml}$. This was in concordance with the study by Jerome et al., in 2006 demonstrated that the comparison of the Vitek-2 Colistin susceptibility test to the broth micro dilution reference test showed a high level of agreement. The resistant subpopulations of the A. baumannii isolates were detected in the Vitek-2, as well as in the other methods for Colistin susceptibility testing in their study. A Similar observation was made by T. Y. Tan et al., in 2006where they found an essential agreement between Vitek-2 and agar dilution in $75 \%$ of the tests which was again in concordance with our study. The isolates used in their study were also nosocomial and hence unlikely to be representative of the general population. No Colistin-resistant Acinetobacter spp. was detected in their study as well as ours therefore it has not been possible to draw definite conclusions concerning the efficacy of the Vitek-2 system in detecting Colistin resistance in this genus. In our Study, no major or Very major errors (VMEs) were noted in the susceptibility testing by Vitek2 , and this was in concordance with the study by Dafopoulou et al., in 2015that was able to identify all Colistin-resistant isolates, with MEs being observed for only two Acinetobacter baumannii. They suggested that Vitek-2 appeared to be a useful method for rapid detection of Colistin resistance, as it exhibited excellent Concordance with Broth microdilution. Our study was also in concordance with a study by Lee et al., in 2013 which demonstrated that only $0.9 \%$ VMEs and no MEs in the 13 isolates of Colistin-resistant Acinetobacter species by Vitek2. Among the three test methods used in our study the most cost effective was Broth micro dilution (Rs 20) where as Vitek 2 was found to be the most expensive (Rs 243). Broth micro dilution, however was the most time consuming (22.1 hrs) as compared to the Vitek 2 (8.7 hrs) which was the least time consuming of the three test methods. The limitation of our study is that no Colistin-resistant Acinetobacter baumannii isolates were found and hence could not be tested. Among commercial methods, E-test is convenient and widely applied in clinical laboratories. Most studies have demonstrated the concordance of the E-test to be as high as $90-100 \%$ and have suggested it as a reliable and useful alternative to the dilution methods (Boyen et al., 2010; Bolmstrom et al., 2005) On the other hand when Tan et al in 2006 compared the E-test with agar dilution for Colistin susceptibility, they reported one major error (2\%) when testing Acinetobacter spp. They concluded that results obtained by E- test may require confirmation by a standard MIC susceptibility testing method. In our 
study, E-test showed 99\% Categorical Agreement with the reference method in Acinetobacter spp. The Categorical agreement (CA) of Vitek-2 as compared to the reference method was $100 \%$ in our study and hence could be a reliable method of Colistin Susceptibility testing for Acinetobacter spp. Similar results were provided by Jerome et al., in 2006 who concluded that The Vitek-2 Colistin susceptibility test was reliable and easy-touse tool to determine susceptibility to Colistin in isolates of genera that were known not to exhibit hetero resistance (Jerome et al., 2007).This was also in concordance to the study by A M SimoonsSmit et al who concluded that the Vitek system was highly accurate in the identification and antibiotic susceptibility testing of clinically important Gram negative bacteria. Tan et al in 2007, however, considered the Vitek-2 Colistin susceptibility test to be an unreliable method. ${ }^{[86]}$ In contrast, the Vitek-2 Colistin susceptibility test performed well in our study. We found a high level of agreement with the reference broth micro dilution method. The Reproducibility and reliability of the E-test for susceptibility testing of Colistin MICs was, hence, found to be as good as that by Vitek-2 testing in our study. The Broth Micro dilution test was found to be the cheapest of the three tests. Luber, Petra et al in their study in 2003 reported the usefulness of the broth micro dilution method for determination of the antibiotic susceptibility patterns of Campylobacter spp. which they confirmed as reasonably priced. The Vitek-2 test system was found to be the least cost effective among the three and this was in concordance with a study by Berke, I., and Tierno, P. M in 1996 who compared the efficacy and costeffectiveness of BIOMIC VIDEO and Vitek antimicrobial susceptibility test systems for use in the clinical microbiology laboratory. They concluded that although the Vitek system allowed rapid, automated testing of antibiotic substances the system as well as the associated service fees could prove to be expensive and limited in some ways (Berke and Tierno, 1996). In our study the Vitek test system was found to be the most time effective (Eigner and Schmid, 2005) while the broth micro dilution was the least time effective of the three test methods. After comparing the two test methods against the reference broth micro dilution method in terms of the various parameters studied and comparing our results with the previous studies, it could be concluded that the best reference method for testing susceptibility to the polymyxins still remains to be defined, however, in routine clinical practice in most regions worldwide, where a reference method can hardly be implemented, the interpretation of Colistin susceptibility should preferably be based on results of automated systems such as Vitek2 or the E-test. Although, our study suggests a good concordance between Vitek and micro broth dilution with $E$ test not significantly lagging behind however there are many un resolved questions regarding polymyxin susceptibility testing( $\mathrm{Li}$ et al., 2005)In addition, there is no consensus regarding the interpretative breakpoints for polymyxin susceptibility; e.g., the CLSI specifies susceptibility breakpoints of $<4 \mathrm{mg}$ $\Lambda$ for Acinetobacter spp., whereas the guidelines of the British Society for Antimicrobial Chemotherapy specify a susceptibility breakpoint of $<8 \mathrm{mg} \Lambda$. Keeping in mind, the increased use of Polymyxins in the treatment of multi resistant Gram-negative nosocomial infections, further studies may be required to evaluate the best method for susceptibility testing for these compounds.

\section{CONCLUSION}

We compared two antibiotic susceptibility test methods, The Vitek-2 and the E-test, against the reference broth micro dilution method in terms of the various parameters such as Reproducibility, reliability, cost and time effectiveness and after comparing our results with the previous studies, it could be concluded that the best reference method for testing 
susceptibility to the polymyxins still remains to be defined. However, in routine clinical practice in most regions worldwide, where a reference method can hardly be implemented, the interpretation of Colistin susceptibility should preferably be based on results of automated systems such as Vitek2 or the E-test. The micro broth Dilution method remains the most reliable and reproducible, however most tedious and time-consuming method. Colistin remains a very effective, least resisted drug for MDR Acinetobacter species as compared by all the three methods. The judicious and careful use of Colistin in our institutional settings is the probable reason that Colistin resistance has not significantly affected our inpatients i.e. 99 to $100 \%$ evidence as suggested by our study.

\section{Acknowledgement: NA}

\section{Conflict of Interest: None}

\section{Source of Funding: None}

\section{Ethical Approval: Approved}

\section{REFERENCES}

1. Ah YM, Kim AJ, Lee JY. 2014. Colistin resistance in Klebsiella pneumoniae. Int $\mathrm{J}$ Antimicrob Agents 44:8-15. doi:10.1016 /j.ijantimicag.2014.02.016.

2. Arroyo LA, García-Curiel A, PachónIbañezME,LlanosAC,Ruiz M, Pachón J, Aznar J. 2005. Reliability of the E-test method for detection of Colistin resistance in clinical isolates of Acinetobacter baumannii. J ClinMicrobiol 43:903-905. doi:10.1128/JCM .43.2.903-905.2005.

3. Berke, I. and TiernoJr, P.M., 1996. Comparison of efficacy and costeffectiveness of BIOMIC VIDEO and Vitek antimicrobial susceptibility test systems for use in the clinical microbiology laboratory. Journal of Clinical Microbiology, 34(8), pp.1980-1984.

4. Bolmstrom KA, Mills K, Vidh P, Giske C. Quantifying synergy between Colistin and rifampicin against carbapenem resistant Acinetobacter with a novel combination gradient. 45 thInterscience Conference Antimicrobial Agents Chemotherapy.
Washington DC: American Society for Microbiology; 2005.

5. Boyen, F., Vangroenweghe, F., Butaye, P., De Graef, E., Castryck, F., Heylen, P., Vanrobaeys, M. and Haesebrouck, F., 2010. Disk prediffusion is a reliable method for testing Colistin susceptibility in porcine E. coli strains. Veterinary microbiology, 144(34), pp.359-362.

6. Cai, Y., Chai, D., Wang, R., Liang, B. and Bai, N., 2012. Colistin resistance of Acinetobacter baumannii: clinical reports, mechanisms and antimicrobial strategies. Journal of antimicrobial chemotherapy, 67(7), pp.1607-1615.

7. Dafopoulou, K., Zarkotou, O., Dimitroulia, E., Hadjichristodoulou, C., Gennimata, V., Pournaras, S. and Tsakris, A., 2015. Comparative evaluation of Colistin susceptibility testing methods among carbapenem-nonsusceptible

Klebsiellapneumoniae and Acinetobacter baumannii clinical isolates. Antimicrobial agents and chemotherapy, 59(8), pp.46254630.

8. Doern, G.V., Vautour, R., Gaudet, M. and Levy, B., 1994. Clinical impact of rapid in vitro susceptibility testing and bacterial identification. Journal of clinical microbiology, 32(7), pp.1757-1762.

9. Eigner, U., Schmid, A., Wild, U., Bertsch, D. and Fahr, A.M., 2005. Analysis of the comparative workflow and performance characteristics of the VITEK 2 and Phoenix systems. Journal of clinical microbiology, 43(8), pp.3829-3834.

10. Gales, A.C., Reis, A.O. and Jones, R.N., 2001. Contemporary assessment of antimicrobial susceptibility testing methods for polymyxin B and Colistin: review of available interpretative criteria and quality control guidelines. Journal of clinical microbiology, 39(1), pp.183-190.

11. Goldstein, F.W., Ly, A. and Kitzis, M.D., 2007. Comparison of Etest with agar dilution for testing the susceptibility of Pseudomonas aeruginosa and other multidrug-resistant bacteria to Colistin. Journal of antimicrobial chemotherapy, 59(5), pp.1039-1040.

12. Hindler, J.A. and Humphries, R.M., 2013. Colistin MIC variability by method for contemporary clinical isolates of multidrugresistant Gram-negative bacilli. Journal of clinical microbiology, 51(6), pp.1678-1684. 
13. Huidrom S, Girish N, Rajendran R. Isolation and study of antimicrobial susceptibility and resistance patterns of Acinetobacter spp. from Intensive Care Unit of a tertiary care hospital in Bengaluru. International Journal of Advanced Research. 2015; 3:1121-1125.

14. Jerome R. Lo-Ten-Foe1, Anne Marie G. A. de Smet2, Bram M. W. Diederen1, †, Jan A. J. W. Kluytmans1,3 and Peter H. J. van Keulen1. Comparative Evaluation of the VITEK 2, Disk Diffusion, Etest, Broth Micro dilution, and Agar Dilution Susceptibility Testing Methods for Colistin in Clinical Isolates, Including Hetero resistant Enterobacter cloacae and Acinetobacter baumannii Strains ${ }^{\nabla}$

15. Jia, P., Xue, Y.J., Duan, X.J. and Shao, S.H., 2011. Effect of cinnamaldehyde on biofilm formation and sarA expression by methicillin-resistant Staphylococcus aureus. Letters in applied microbiology, 53(4), pp.409-416.

16. Jossart, M.F. and Courcol, R.J., 1999. Evaluation of an automated system for identification of Enterobacteriaceae and nonfermenting bacilli. European Journal of Clinical Microbiology and Infectious Diseases, 18(12), pp.902-907.

17. Kandeel et al/ Detection of Colistin susceptibility by four different methods, Egyptian Journal of Medical Microbiology Volume 25 / No. 2 / April 2016 17-23.

18. Karaiskos, I. and Giamarellou, H., 2014. Multidrug-resistant and extensively drugresistant Gram-negative pathogens: current and emerging therapeutic approaches. Expert opinion on pharmacotherapy,15(10), pp.1351-1370.

19. Karvanen, M., Malmberg, C., Mohamed, A., Lagerbäck, P., Friberg, L.E. and Car, O., 2011, September. Colistin is extensively lost during normal experimental conditions. In Fifty-first Interscience Conference on Antimicrobial Agents and Chemotherapy, Chicago, IL.

20. Landman, D., Georgescu, C. and Martin, D.A., 2008. Quale J. Polymyxins Revisited. Clin. Microbiol. Rev, 21, pp.449-465.

21. Lee, S.Y., Shin, J.H., Lee, K., Joo, M.Y., Park, K.H., Shin, M.G., Suh, S.P., Ryang, D.W. and Kim, S.H., 2013. Comparison of the Vitek 2, MicroScan, and Etest methods with the agar dilution method in assessing
Colistin susceptibility of bloodstream isolates of Acinetobacter species from a Korean university hospital. Journal of clinical microbiology, 51(6), pp.1924-1926.

22. Ligozzi, M., Bernini, C., Bonora, M.G., De Fatima, M., Zuliani, J. and Fontana, R., 2002. Evaluation of the VITEK 2 system for identification and antimicrobial susceptibility testing of medically relevant gram-positive cocci. Journal of clinical microbiology, 40(5), pp.1681-1686.

23. Lo-Ten-Foe, J.R., de Smet, A.M.G., Diederen, B.M., Kluytmans, J.A. and van Keulen, P.H., 2007. Comparative evaluation of the VITEK 2, disk diffusion, Etest, broth microdilution, and agar dilution susceptibility testing methods for Colistin in clinical isolates, including heteroresistant Enterobacter cloacae and Acinetobacter baumannii strains. Antimicrobial agents and chemotherapy, 51(10), pp.3726-3730.

24. Luber, P., Bartelt, E., Genschow, E., Wagner, J. and Hahn, H., 2003. Comparison of broth microdilution, $\mathrm{E}$ test, and agar dilution methods for antibiotic susceptibility testing of Campylobacter jejuni and Campylobacter coli. Journal of clinical microbiology, 41(3), pp.1062-1068.

25. Nation, R.L., Li, J., Cars, O., Couet, W., Dudley, M.N., Kaye, K.S., Mouton, J.W., Paterson, D.L., Tam, V.H., Theuretzbacher, U. and Tsuji, B.T., 2015. Framework for optimisation of the clinical use of Colistin and polymyxin B: the Prato polymyxin consensus. The Lancet infectious diseases, 15(2), pp.225-234.

26. Roohi, S., Samad, L., Wani, S., Fomda, B.A., Lone, M.S., Ahmad, J., Fatima, A. and Jan, A., 2017. Multidrug Resistant Acinetobacter Infection in Surgical Intensive Care Unit in a Tertiary Care Center in North India. Journal of Contemporary Medical Research, 4(3), pp.621-624.

27. Sader, H.S., Farrell, D.J., Flamm, R.K. and Jones, R.N., 2014. Antimicrobial susceptibility of Gram-negative organisms isolated from patients hospitalized in intensive care units in United States and European hospitals (2009-2011).Diagnostic microbiology and infectious disease, 78(4), pp.443-448.

28. Simoons-Smit, A.M. and Maclaren, D.M., 1994. Comparison of Vitek and Cobas Micro systems with a semiautomated 
Nazir Ahmad Var et.al. Comparison of Colistin MIC by Microbroth dilution, E-test and Vitek-2, in isolates of Acinetobacter spp. isolated from bloodstream infections

conventional microsystem for identification and susceptibility testing of gram-negative bacilli. Journal of clinical pathology, 47(1), pp.71-75.

29. Tan, T.Y. and Ng, S.Y., 2007. Comparison of Etest, Vitek and agar dilution for susceptibility testing of Colistin. Clinical microbiology and infection, 13(5), pp.541544.

30. Walkty, A., DeCorby, M., Nichol, K., Karlowsky, J.A., Hoban, D.J. and Zhanel, G.G., 2009. In vitro activity of Colistin (polymyxin E) against 3,480 isolates of gram-negative bacilli obtained from patients in Canadian hospitals in the CANWARD study, 2007-2008. Antimicrobial agents and chemotherapy, 53(11), pp.4924-4926.

How to cite this article: Var NA, Wani NA, Ahmad SK. Comparison of Colistin MIC by Microbroth dilution, E-test and Vitek-2, in isolates of Acinetobacter spp. isolated from bloodstream infections. International Journal of Research and Review. 2021; 8(12): 218-233. DOI: https://doi.org/10.52403/ijrr.20211228 